\title{
LA EXPERIENCIA DE E-OCIO DE LOS JÓVENES
}

\author{
THE E-LEISURE EXPERIENCE OF YOUTH
}

Ana Viñals Blanco ${ }^{1}$

\section{Resumen}

La era digital y las tecnologías digitales han transformado el ámbito del ocio, entendido en este artículo como experiencia de desarrollo humano. Internet ha posibilitado nuevas prácticas de ocio que están teniendo especial auge entre los jóvenes nativos interactivos, quienes al nacer rodeados de pantallas sienten el espacio online como una parte esencial de su cotidianidad. El objetivo de este artículo es describir los rasgos de e-ocio de los jóvenes y su percepción al respecto, a través de un estudio que se fundamenta en el análisis e interpretación del discurso extraído del desarrollo de nueve grupos de discusión y diez entrevistas en profundidad con jóvenes de entre 16 y 18 años de Bizkaia. Una valoración en su conjunto positiva, pero de carácter superficial. El artículo se distribuye en tres partes. En primer lugar, se explica la transformación que ha sufrido la esfera del ocio en la era digital y se exponen las características de la generación digital y los rasgos de su e-ocio; en segundo lugar, se presentan los resultados de la investigación que recogen las percepciones que los jóvenes han manifestado; y, por último, aportamos una interpretación de los resultados desde la perspectiva experiencial del ocio.

Palabras clave: e-ocio; jóvenes; internet; TIC

\section{Abstract}

The digital era and the digital technologies have transformed the field of leisure, understood in this article as an experience of human development. Internet has enabled new leisure practices that are having special success among young interactive natives, who feel the online space as an essential part of everyday life because they are surrounded by screens since their birth. The aim of this article is to describe the features of e-entertainment of young people and their perception about it, through a study based on the analysis and interpretation of the extracted speech development of nine discussion groups and ten in-depth interviews with young people between 16 and 18 years of Bizkaia. A positive assessment in its whole, but superficial character. The article is divided in three parts. First, the transformation suffered by the sphere of leisure in the digital era and the characteristics of the digital generation and the features of their e-leisure are explained; secondly, the research results that reflect the perceptions that young people have expressed are presented and, finally, we provide an interpretation of the results from the experiential perspective of leisure.

Keywords: e-leisure; young people; internet; ICT

Fecha de recepción: 23 de octubre de 2015

Fecha de aprobación: 4 de marzo de 2016

Para citar este artículo:

Viñals, A. (2016). La experiencia de e-ocio de los jóvenes. Lúdica Pedagógica, (23), 93-103.

1 Dra. Ocio y Desarrollo Humano. Instituto de Estudios de Ocio. Universidad de Deusto. Máster en Organización de Congresos, Eventos y Ferias y licenciada en Publicidad y Relaciones Públicas. Correo electrónico: ana.vinals@deusto.es 


\section{INTRODUCCIÓN}

Este artículo se enmarca en la actual sociedad digital en la que los hábitos y estilos de vida se han visto transformados por el desarrollo imparable de las tecnologías de la información y la comunicación (en adelante TIC) e Internet. Una era digital del "todo conectado", en la que la tecnología ha pasado a ocupar un lugar central en una gran parte de los ámbitos de actuación humana, entre ellos, la esfera del ocio, ámbito central de análisis de este artículo que no ha podido quedarse al margen de la digitalización de la vida. Las tecnologías digitales no solo han posibilitado el disfrute de nuevos ocios ligados al espacio online (redes sociales virtuales, videojuegos online, comunidades virtuales, blogs, etc.), sino que, además, han modificado la manera en que las personas, en general, y los jóvenes en particular, experimentan y disfrutan de su ocio.

La generación de jóvenes interactivos, nacidos en plena explosión de lo digital, posee unas características que les diferencian de sus progenitores. Son jóvenes multitarea que, inmersos en una cultura digital y audiovisual, están acostumbrados a vivir en una acción constante, a recibir recompensas inmediatas y a estar conectados las 24 horas del día. Además, al nacer rodeados de aparatos tecnológicos y pantallas, tienen cierta facilidad en el manejo de las Tic e Internet, lo que no significa que sean competentes digitales. Para ellos Internet es básicamente una herramienta y espacio en el que se divierten, se relacionan y se informan, esto es, apenas reparan en su verdadero potencial como útiles de aprendizaje, participación y empoderamiento ciudadano.

Conscientes del papel que Internet ocupa hoy en la vida de los jóvenes, el objetivo de este artículo es describir los rasgos del ocio conectado ${ }^{2}$ de los jóvenes, con especial hincapié en analizar la percepción que los jóvenes, de entre 16 y 18 años de Bizkaia (Euskadi), tienen de su ocio en $\mathrm{red}^{3}$. Un estudio de marcado carácter cualitativo, en el que se ha analizado el discurso, a través del

2 A lo largo del artículo utilizaremos indistintamente los términos ocio conectado, ocio en red, e-ocio, ocio online, etc. para describir el ocio que necesita de conexión a Internet para disfrutarse.

3 Este análisis se deriva la tesis doctoral titulada "Ocio conectado: la experiencia de e-ocio de los jóvenes (16-18 años) de Bizkaia" que la autora del articulo acaba de depositar. Un estudio cuyo objetivo ha sido analizar, desde la perspectiva experiencial del ocio, las características de e-ocio de los jóvenes de entre 16 y 18 años de Bizkaia de centros educativos de rasgos diferentes, a partir de la elaboración de 9 grupos de software de datos cualitativos Atlas.ti versión 7.5.6, de un total de 88 jóvenes de entre 16 y 18 años de Bizkaia, participantes de nueve dinámicas grupales de grupos de discusión y diez entrevistas en profundidad.

El artículo se ha estructurado en tres apartados. En primer lugar, y a modo de marco teórico, se explica la transformación que ha sufrido la esfera del ocio en la era digital y se exponen las características de la generación digital y los rasgos de su e-ocio; en segundo lugar, se presentan los resultados de la investigación que recogen las percepciones que los jóvenes han manifestado de su ocio en red y, por último, aportamos una interpretación de los resultados desde la perspectiva experiencial del ocio.

\section{EL OCIO EN LA ERA DIGITAL}

La interactividad, la hipertextualidad y la conectividad (Kerckhove, 1999) son rasgos propios de la red que han influido en la manera de comunicarnos, socializarnos, informarnos, hacer la compra, ver la televisión, trabajar, etc. Esto es, las tecnologías digitales han transformado las experiencias de ocio.

Las dimensiones del tiempo y el espacio, constitutivas de la vida y la cultura humanas, han cambiado por completo. Los espacios y las localidades se han ido poco a poco desprendiendo de su significado cultural, histórico y geográfico, reintegrándose en redes funcionales o en collage de imágenes que provocan espacios de flujos. El tiempo se ha reprogramado, hecho continuo y tornado atemporal (Castells, 1997). Todo ello ha influido en el modo de vivir el ocio tradicional, pero, además, ha propiciado el desarrollo de nuevos ocios ligados al uso de Internet y experimentados en el ciberespacio.

Nos encontramos ante una clara digitalización de las experiencias de ocio. Dicho de otro modo, nos hallamos ante la configuración de un nuevo paradigma de experiencia (Cuenca, 2010) que soporta características específicas propias de los espacios en red. Influencias externas que han dado lugar a la configuración de nuevas actividades de ocio propias de la era digital que necesitan de una correcta educación que trasmita a la ciudadanía, en general, y a los jóvenes, en particular, los

discusión y 10 entrevistas en profundidad. Director: Manuel Cuenca Cabeza; Codirector: Jaime Cuenca Amigo. 
conocimientos, habilidades y actitudes necesarias para poder disfrutar y aprovechar las oportunidades que este "nuevo" espacio proporciona.

A día de hoy, podemos comprobar que no existe un tiempo exclusivo para el disfrute del ocio, esto es, una parcela destinada al ocio fuera de la jornada laboral o el periodo referido a la vacaciones, sino que, como plantea San Salvador del Valle (2009), el ocio ha pasado a concebirse como un verdadero fast-ocio que implica una desaforada búsqueda del aprovechamiento al límite del tiempo percibido como bien escaso. Partiendo de la distinción entre sociedad sólida y líquida, expuesta por Bauman (1999), se puede afirmar que el ocio actual es un ocio líquido (Cuenca, Bayón y Madariaga, 2012). Un tipo de ocio que ha adoptado unos rasgos basados en el cambio constante, la aceleración, el disfrute breve de las cosas, la transitoriedad, la fragilidad de los vínculos humanos y la incertidumbre persistente. Un ocio asentado en una sociedad en red en perpetuo movimiento, donde lo que realmente importa es estar en permanente conexión con los demás.

En este sentido, debido a que la ubicuidad ${ }^{4}$ que posibilitan estos entornos en red encaja a la perfección con el modelo y estilo de ocio que hoy impera, cada vez más los espacios virtuales se conciben como espacios de ocio. Un e-ocio simultáneo y atemporal propio de una cultura de la virtualidad real de este mundo digital que, debido al desarrollo imparable de los smartphones, ha posibilitado que el ocio no se disfrute exclusivamente en contextos informales y apartados del horario laboral, sino que pase a inmiscuirse a lo largo de todos los tiempos sociales que conforman el día.

Tal y como argumenta Igarza (2009), el ocio actual se entremezcla entre los bloques productivos (de trabajo), los tiempos de espera (dentista, supermercado, etc.) o los desplazamientos (metro, tren, coche, etc.). Por esta razón, Igarza define el ocio de la sociedad de la información como un ocio más interactivo, más selectivo, menos duradero y basado en micro-guiones, micro-estructuras y micro-contenidos, esto es, un ocio intersticial que ha transformado al ciudadano del siglo XxI en un ciudadano wifi, que en ocasiones no puede prescindir de la relación con las nuevas tecnologías en su vida diaria.

4 Que está presente a un mismo tiempo en todas partes (RAE). Última consulta: 21/10/2015 http://dle.rae.es/?w=ubicuo\&m= form\&o=h
En estas Burbujas de ocio (Igarza, 2009) el entretenimiento, el placer, la distensión, el esparcimiento y la diversión es lo que prima. En definitiva, un tipo de ocio que no sería posible sin el avance hacia la creación de soportes tecnológicos económicamente más accesibles, ágiles, dinámicos, de fácil acceso y uso para la ciudadanía en su conjunto. Una industria del ocio digital que ha provocado un mayor incremento en el consumo de contenidos digitales y que ha impulsado la digitalización de las prácticas de ocio tradicionales y los "nuevos" e-ocios. Ahora bien, ¿qué entendemos por ocio digital y e-ocio?

De manera general, el ocio digital es el tipo de ocio que se liga a las infinitas oportunidades que ofrecen las tecnologías digitales. Por ocio digital comprenderíamos, como se observa en la figura 1, por un lado, el ocio tradicional que se ha digitalizado y ha sufrido la influencia de la tecnología y, por otro lado, el ocio que se practica en red y necesita de conexión para su disfrute (e-ocio). Una tipología de ocio digital que hemos dividido en dos términos: ocio tradicional digitalizado y nuevos ocios.

La anterior distinción la realizamos con base en la noción de estar o no conectado. Por ocio tradicional digitalizado nos estaríamos refiriendo a las actividades de ocio hasta el momento practicadas que de alguna manera se han tecnologizado, por ejemplo, la lectura y su paso de formato papel al libro electrónico; y por e-ocio consideraríamos a todo el conjunto de actividades de ocio en línea (redes sociales virtuales, blogs, wikis, foros, comunidades virtuales, etc.) que necesitan de ocio para su disfrute. Nuevos ocios que surgen del uso de todo un conjunto de servicios y aplicaciones en línea que facilitan la edición, la publicación y el intercambio de información entre los propios usuarios. A saber, blogs, wikis, redes sociales, comunidades virtuales y un sin fin de servicios que conforman el universo $2.0 \mathrm{y}$ que facilitan la colaboración entre los usuarios. En otras palabras, un ocio digital que se cimenta en conexiones.

En definitiva, cuando distinguimos entre espacios de ocio físicos y virtuales simplemente se pretende hacer referencia a la confección de nuevos espacios y contextos de ocio que han posibilitado nuevas oportunidades de disfrutar del tiempo libre. Es decir: 
Figura 1. Tipología del concepto de ocio digital

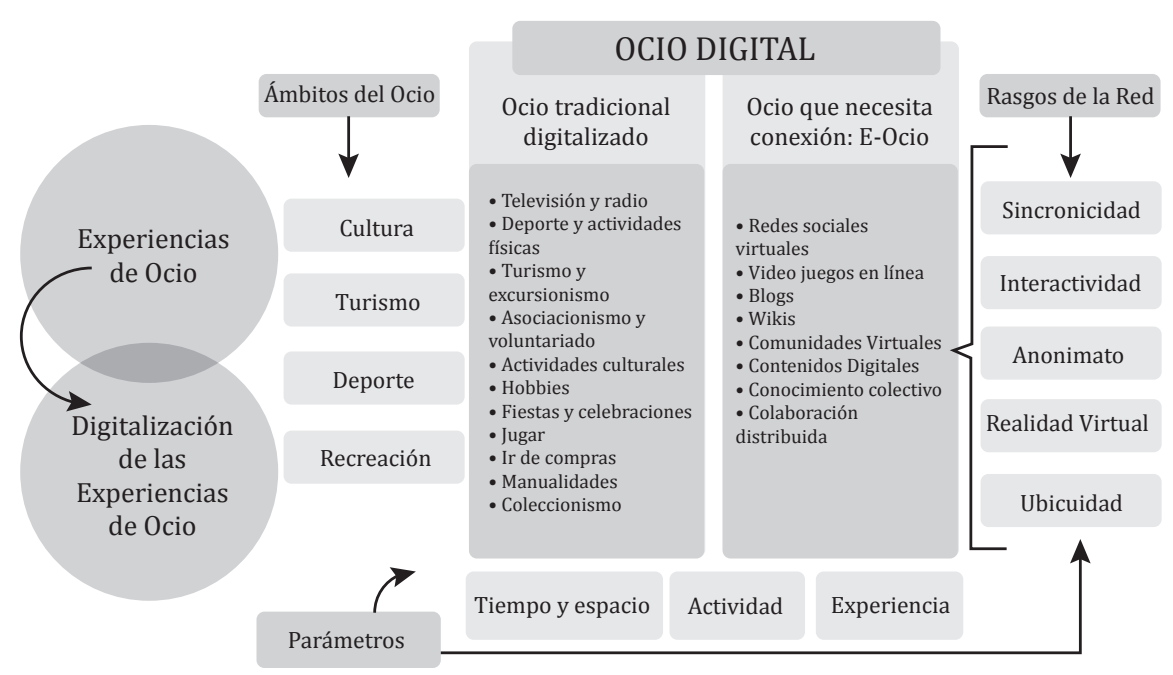

Fuente: Elaboración propia (basada en las ideas de Nimrod y Adoni, 2012).

Interpretamos el ocio digital en Internet no como un estar conectado (ocio online) frente a no conectado (ocio offline), sino como seguir conectado al mundo social digitalmente construido, no como un ocio virtual frente a un ocio real, sino como un nuevo espacio donde continuar las relaciones sociales. (García, López y Samper, 2012, p. 397).

\section{JÓVENES Y E-OCIO}

Estas prácticas de ocio conectado que acabamos de definir están teniendo especial auge entre los jóvenes nativos interactivos. Una generación que al nacer rodeada de pantallas siente el espacio online como una parte esencial de su cotidianidad. Tanto los jóvenes nacidos en la era digital como sus estilos de ocio han adquirido unos rasgos propios. De hecho, han sido bautizados de muy diversas maneras: generación@ (Feixa, 2002), nativos digitales (Prensky, 2010), generación net (Tapscott, 1998), generación interactiva (Bringué y Sádaba, 2009), etc. Un sin fin de nociones que independientemente de su originalidad o acierto hacen referencia a un grupo de personas nacidas en torno a la década de los 90, rodeados de tecnologías digitales, con formas de relación similares y preocupaciones, intereses u opiniones en muchas ocasiones comunes; jóvenes que están creciendo en un contexto social, cultural y educativo distinto, especialmente por la presencia continua y constante de la tecnología, y que presentan rasgos distintivos respecto a otras generaciones. ¿Cómo es la generación de jóvenes interactivos?
Los jóvenes de la era digital se caracterizan por ser jóvenes multitarea, es decir, pueden llevar a cabo varias tareas al mismo tiempo. Viene siendo muy común observar a jóvenes que mientras descargan música de Internet, chatean con sus amigos a través del teléfono móvil, consultan la biblioteca virtual instalada en su ordenador portátil, hacen los deberes y ven la televisión al mismo tiempo. Son jóvenes interactivos para quienes hacer varias cosas a la vez se ha convertido en una forma de vida e incluso una necesidad. Jóvenes que bajo la existencia de una sociedad infoxificada (exceso de información) no realizan un análisis crítico de dicha abundancia de datos. Lo que conlleva que su atención resulte cada vez más diversificada, aunque disminuida con respecto a cada estímulo.

El segundo rasgo que les define es que son jóvenes $100 \%$ audiovisuales, esto es, viven inmersos en una cultura digital donde predomina el poder de la imagen y el sonido por encima de la palabra escrita. No es de extrañar que los nativos interactivos prefieran los gráficos, al discurso escrito. De esta manera, es muy usual que hagan uso del texto únicamente para ilustrar la imagen, y tan solo en caso de que no comprendieran esta acudirían a consultar el texto.

También son jóvenes que se sienten atraídos por la acción constante (Prensky, 2010), que desarrollan mentes hipertextuales y navegan por Internet saltando de una información a otra. Son una generación click, multitarea, que no reflexiona sobre sus propias acti- 
tudes y conductas y que opina que lo importante es hacer, más que hacer bien. Por lo tanto, y al encontrarse en constante acción, es lógico que los libros de texto convencionales les resulten aburridos y les cueste mantener una lectura pausada. Al respecto, Carr (2011) opina que navegar por la red fomenta el picoteo rápido y distraído de pequeños fragmentos de información de muchas fuentes distintas. De ahí que, bajo el argumento relacionado con que la ética de Internet es una ética industrial, de la velocidad y la eficiencia, el autor afirme que Internet hace que disfrutemos de ser superficiales. Una idea con la que los neurocientíficos Small y Vorgan (2008) no está del todo de acuerdo, ya que considera que el uso de Internet tiene resultados positivos para el funcionamiento del cerebro, siempre y cuando su uso no sea excesivo.

Al mismo tiempo, los nativos interactivos presentan una capacidad de actualización constante, están acostumbrados a pedir y recibir. Es por ello que precisan de una gratificación instantánea con recompensas materiales frecuentes. Al estar envueltos en una sociedad consumista, necesitan saber para qué les sirve lo que van a realizar en el mismo instante en el que se proponen realizarlo. Por todo ello, valores como el esfuerzo y la constancia quedan a un lado, sucumben ante la filosofía del "aquí y ahora".

Son jóvenes que, al nacer en un mundo digitalizado, han adquirido cierta fluidez en el manejo de este tipo de tecnologías sociales. Utilizan con total naturalidad cámaras de fotos y vídeo digitales, navegan por la red con total soltura, juegan online, y se comunican a través de todo tipo de redes multimedia, etc. Esto, sin embargo, no determina que realicen un uso correcto y un buen aprovechamiento del potencial positivo de las mismas. Se les tiende a catalogar como prosumidores, esto es, consumidores y productores de contenido, no obstante, como describimos a continuación, los jóvenes, en general, hacen un uso utilitarista y pasivo de la tecnología. Básicamente, las conciben como herramientas de comunicación, socialización, entretenimiento y para pasar el rato, es por ello que, a priori, no las identifican como potentes instrumentos para el aprendizaje y su desarrollo personal.

En términos generales, el estilo de ocio de los jóvenes de la era digital adopta un componente tecnológico alto, valorado positivamente por los propios usuarios. El orde- nador y el teléfono móvil son dispositivos tecnológicos que se han domesticado ya en sus vidas, y el $79 \%$ de los jóvenes se conecta a ellos todos o casi todos los días. A nivel de Euskadi y según el Observatorio Vasco de Juventud (en adelante ovJ, 2009), el 76,5 \% de la juventud vasca afirma que, de media, tiene tres o más horas de tiempo libre al día durante los días de labor. De las cuales entre una y dos horas las ocupan conectados a Internet.

Las cuatro actividades de e-ocio más practicadas por los jóvenes son (Instituto de Juventudes de España, INJUVE, 2012; Observatorio Nacional de las Telecomunicaciones y de la Sociedad de la Información, ONTSI, 2012; Observatorio Vasco de la Juventud, ovJ, 2012, etc.): 1. Buscar información; 2. Recibir o enviar mensajes de correo electrónico; 3. Utilizar las redes sociales virtuales como Facebook, Twitter o Tuenti, y 4. Descargar archivos, documentos, música, vídeo, software, etc.

Atendiendo a la utilidad personal de la red, el ovj (2012) muestra que el entretenimiento (60\%) es la primera opción con la que los jóvenes identifican la red, seguida del interés por mantenerse en contacto con otras personas (54\%), estar informado/a de las noticias de actualidad (37\%) y aprender cosas interesantes (25 \%). Sus contenidos favoritos son la música $(75,4 \%)$, los juegos $(65,8 \%)$ y los deportes (42,5\%). Es decir, el uso que los jóvenes hacen de la red se lleva a cabo desde una perspectiva consumista, pasiva y con el fin principal de informarse, comunicarse, entretenerse y pasar el rato.

Respecto a la valoración de los distintos aparatos tecnológicos, el $91 \%$ de los y las jóvenes de 18-24 años considera el teléfono móvil como "bastante o muy necesario para la vida cotidiana" (Centro Reina Sofía sobre Adolescencia y Juventud, CRS-FAD, 2014). Seguidos de disponer de conexión a Internet (87\%), ordenador personal (82\%), correo electrónico (79\%), conexión a Internet de alta velocidad (61\%) y redes sociales (57 \%). En el mismo sentido, el informe jóvenes 2012 (INJUVE, 2012) muestra cómo la mayoría de las personas jóvenes considera que Internet es "muy o bastante útil" para la vida cotidiana, pero mucho más la disponibilidad de teléfono móvil.

Se puede decir que el uso intensivo de las tecnologías digitales en el tiempo libre de los jóvenes y el éxito de masas de las redes sociales y los smartphones perfila cuatro tipologías de e-ocio: un e-ocio social, un e-ocio lúdico, un e-ocio móvil y un e-ocio transmedia. 
Un ocio digital social en el que predomina el uso de las redes sociales virtuales, un ocio digital móvil que posibilita la conectividad sin límites que ofrecen los smartphone, un ocio digital lúdico ligado al uso de los videojuegos y, por último, un ocio transmedia y multisoporte donde los jóvenes simultanean, en tiempo real, el uso de diferentes dispositivos para acceder a un mismo contenido y generar sus propias narrativas.

En breve, los jóvenes han cambiado, la tecnología forma parte de sus vidas y, por ende, sus estilos de ocio también han variado. A continuación, y más hacia lo concreto, nos centramos en exponer las percepciones que los jóvenes de entre 16 y 18 años de Bizkaia tienen de su ocio conectado. Impresiones personales que hemos extraído del análisis del discurso de ellos mismos como protagonistas a través de la organización de nueve grupos de discusión y diez entrevistas en profundidad. Además de conocer cuantitativamente la realidad del ocio en red de los jóvenes, consideramos que es necesario ahondar en sus motivaciones, opiniones, percepciones, etc. Una realidad subjetiva a la que hemos accedido mediante el uso de técnicas de investigación propias del paradigma simbólico que acto seguido detallamos.

\section{METODOLOGÍA}

El análisis de la realidad puede abordarse desde diversos enfoques paradigmáticos. Todo depende del cariz y el tipo de información que se busque conseguir para su estudio. Dicho de otro modo, todo obedece al objetivo general marcado y al proceso que se ha decidido seguir para su consecución.

En esta ocasión, se buscaba profundizar en el conocimiento del e-ocio de los jóvenes desde la perspectiva experiencial de entender el ocio, es decir, desde la concepción del ocio como "experiencia humana (persona y social) integral, motivada por la vivencia intencional del ocio autotélico (con fin en sí mismo), entendido como derecho humano y ámbito de desarrollo, al que se accede mediante la formación" (Cuenca, 2014, p. 66). Un modo particular de pensar y analizar el fenómeno del ocio que ha sido defendido en los últimos 25 años por el equipo oficial y multidisciplinar del Instituto de Estudios de Ocio (IEO) de la Facultad de Ciencias Sociales y Humanas de la Universidad de Deusto.
En este sentido, este estudio se ha realizado desde el paradigma simbólico, también denominado hermenéutico, cualitativo, fenomenológico, interpretativo, microetnográfico o humanístico-etnográfico. Una perspectiva de investigación que contempla la sociedad como una realidad que se crea y se mantiene a través de interacciones simbólicas y pautas de comportamiento. Mientras en el paradigma positivista el interés se centra en la búsqueda de nuevos conocimientos y su generalización, desde el paradigma interpretativo se estudian los fenómenos de carácter social para comprender la realidad circundante en su carácter específico.

Se trata de un planteamiento general de investigación que parte de concebir la existencia de realidades múltiples. Por lo que se focaliza la atención en la descripción de lo individual, lo distintivo y lo particular del hecho que se estudia. La pretensión principal no es establecer regularidades o establecer generalidades a partir de datos estadísticos, "sino que más bien se considera que los postulados de una teoría son válidos únicamente en un espacio y tiempo determinados" (González, 2013, p. 130).

La perspectiva metodológica seleccionada para el estudio determina las técnicas de recogida de información que deben emplearse. En esta ocasión se han utilizado las dinámicas de grupos de discusión y las entrevistas personales en profundidad. Los grupos de discusión o grupos focales se definen como una discusión cuidadosamente diseñada para obtener las percepciones sobre una particular área de interés. Es una técnica no directiva que tiene por finalidad la producción controlada de un discurso por parte de un grupo de sujetos que son reunidos, durante un espacio de tiempo limitado, a fin de debatir sobre determinado tópico propuesto por el investigador (Krueger, 1991). Se trata de establecer y facilitar la discusión y no de entrevistar al grupo, con base en un guión de discusión previamente diseñado. Las entrevistas en profundidad se han utilizado para seguir adentrándonos en el tema, a través de la comprensión individual. Dicho de otro modo, se ha ido paso a paso construyendo minuciosamente la experiencia individual.

El criterio de selección muestral no tenía como propósito representar la población joven, por lo que nos hemos basado en una muestra intencional, que representase la amplitud, variedad e integración de las diversas realidades que convergen en el objeto estudiado, esto es, 
que abarcase los diferentes puntos de vista. Los sujetos objeto de estudio de esta investigación han sido jóvenes de entre 16 y 18 años de Bizkaia y para localizarlos se ha decidido acudir a los centros educativos, en concreto, se ha contactado con los cursos de primero y segundo de bachillerato. Para elegir a los centros se han tomado en consideración estas tres variables: la naturaleza del centro (privado, público o concertado), el empleo o no de metodologías de enseñanza innovadoras o herramientas tecnológicas (móviles, tablets, Ipads, etc.), y el nivel de madurez Tic del centro (básico, medio y avanzado). ${ }^{5}$ Para la selección de los diez jóvenes entrevistados se han seguido los diez perfiles de usuarios de Internet que se determinan en el estudio de Horrigan (2009), ${ }^{6}$ desarrollado en el centro de investigación estadounidense Pew Research Center.

Previa autorización de los informantes, la información cualitativa recolectada y producida por los propios jóvenes participantes en los grupos de discusión y entrevistas en profundidad se ha registrado mediante grabación de audio. Tras la grabación, los datos han sido transcritos utilizando el programa de trascripción F4, un programa que nos ha facilitado y agilizado la transcripción de las conversaciones de audio a una mayor velocidad. Una vez transcritos los datos, se han analizado con el software de análisis cualitativo Atlas. ti 7, versión 7.5.6. Este último nos ha permitido, por un lado, asociar códigos o etiquetas con los fragmentos de texto y audio, y, por otro lado, buscar códigos de patrones y clasificarlos (Lewis, 2004; Hwang, 2007). En esta ocasión se ha seguido una estrategia de categorización mixta, deductiva-inductiva, y una codificación abierta $\mathrm{y}$ en lista.

Tras la recolección, codificación y categorización de los datos se ha procedido al análisis descriptivo de los mismos, esto es, a atribuirles significados. Posteriormente, este análisis se ha interpretado desde diferentes marcos teóricos, esto es, se han elaborado enunciados explicativos de la realidad social.

5 Más información: http://www.hezkuntza.ejgv.euskadi.eus/ contenidos/informacion/dig_publicaciones_innovacion/es_ tecnolog/adjuntos/20_ikt_400/400003c_Pub_EJ_Madurez_ TIC_c.pdf

6 Consultar los perfiles: http://www.pewinternet.org/files/oldmedia/Files/Reports/2009/The_Mobile_Difference.pdf

\section{ANÁLISIS DE LOS RESULTADOS: PERCEPCIÓN DE LOS JÓVENES DE BIZKAIA SOBRE SU E-OCIO}

El análisis del discurso de los jóvenes deja patente una valoración y percepción positiva del componente tecnológico que han adoptado sus estilos de ocio. En concreto, hemos agrupado en seis las representaciones que más repiten en su discurso para definir la manera en que Internet está presente en sus vidas.

Tal y como mostramos en la vista de red de la figura 2 , los jóvenes perciben la red como una herramienta, como un ocio de segunda, como un hábito que forma parte de la vida, como un vicio, como una manera de aislarse y como una distracción.

\section{E-ocio como herramienta}

En primer lugar, los jóvenes consideran que Internet es una herramienta útil y cómoda que les permite estar informados y comunicados en todo momento. Consideran que se trata de una herramienta que les ha posibilitado enriquecer su ocio tradicional, esto es, "estar con los amigos", "hacer deporte" y "pasarlo bien". Valoran de manera positiva las ventajas de poder estar conectado de manera ubicua y así lo expresan:

Figura 2. Percepción de los jóvenes sobre su "e-ocio"

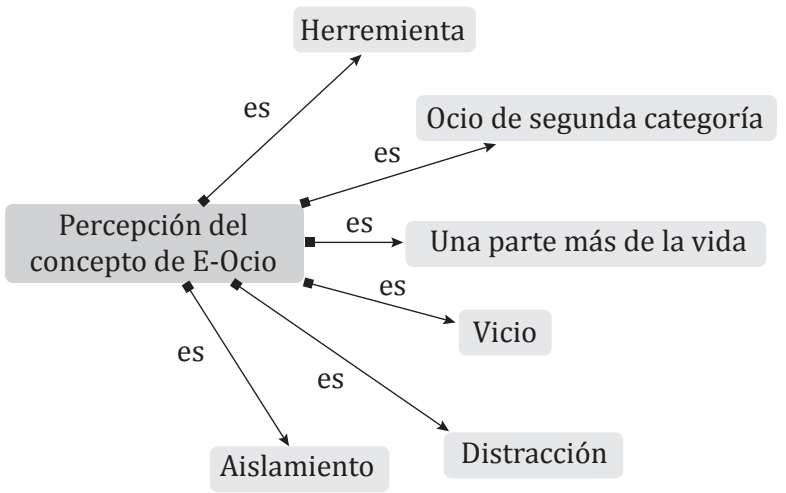

Fuente: Elaboración propia herramienta "vista de red" Atlas.ti

PU3 17 O: Si quieres saber algo puede buscar en Internet y eso también es ocio.

PU1 17 O: Pues que te evades un poco no... porque en clase tienes que estar centrado $\mathrm{y}$ con Internet puedes hablar de lo que quieras con otros compañeros...

C16A: Y es que para quedar con alguien es más fácil mandarle un WhatsApp 
C17 A: Muchas facilidades o por ejemplo para buscar información o conocer a personas de otras partes del mundo

\section{E-ocio como un ocio de segunda}

En segundo lugar, los jóvenes califican el e-ocio como un ocio "de segunda", como "la opción B", como un pasatiempo:

PU3 17 0: Internet es como una segunda opción, si no tienes nada más que hacer. Pero si tienes algo mejor que hacer... ipues no!

PU3 18 O: Te metes en Twitter y dices "venga a ver que se cuece"

\section{E-ocio como una parte más de la vida}

Otra de las maneras que los jóvenes emplean para definir la manera en que Internet está presente en su ocio es afirmando que, en realidad, Internet ha pasado a ser una parte más de la vida. Un hábito, una costumbre, un espacio más en el que poder desarrollarse como personas. Estas afirmaciones son una muestra de la percepción que tienen de Internet como algo omnipresente en nuestra cotidianidad. Al fin y al cabo, y como exponíamos al inicio del artículo, vivimos conectados en una sociedad red, fundamentada en conexiones:

C 19 O: Bueno pero es que al final estas todo el día con el móvil, que si con el WhatsApp...

PU2 17 A: ¡Buf! en la ducha con el móvil, cagando con el móvil, a clase vas como un tonto en plan mirando el móvil y al final parece que lo tienes programado porque sabes en que semáforo te tienes que parar, cuando segundos, luego volver a andar...

\section{E-ocio como vicio}

La necesidad de estar conectado es otro de los términos al que los jóvenes recurren para definir el e-ocio. Los jóvenes manifiestan que Internet, en general, y el WhatsApp, en particular, es imprescindible para poder comunicarse con sus pares. Tal y como se puede comprobar en las citas seleccionadas, disponer de un móvil como conexión a Internet se ha convertido en un recurso de primera necesidad. Hasta el punto en que es tal la dependencia que lo califican como "esclavitud".

PU2 16 A: Yo, de hecho, al principio fui la última casi de la cuadrilla en tener WhatsApp y me lo tuve que poner porque no me enteraba de los planes porque sino no me hubiese puesto...
PU1 17 0: Yo eso lo veo más esclavitud. Yo no tengo Internet en el móvil y de momento no quiero tener, porque yo Internet lo utilizo en casa para estar en Youtube y poner música. Pero en la calle para estar con el WhatsApp ¿Dónde estas? ¿Qué haces? Pues no sé... ¡déjame en paz!

Por último, los jóvenes participantes muestran cierta tendencia a recurrir al uso de expresiones con connotaciones negativas, tales como vicio, aislamiento o distracción. En cuanto al uso de Internet por vicio, los jóvenes lo señalan como una mala costumbre. Un uso indebido y excesivo de la red:

PU2 17 A: Yo es que más que nada estoy tanto en Internet porque tengo vicio, igual tengo que estudiar o así y me meto en Twitter aunque sea para cotillear o cualquier tontería. Pero es como que lo tengo que hacer, si no, no puedo ponerme a estudiar. Aunque esté 5 minutos solo, ya es que me tengo que meter. $\mathrm{Y}$ eso ya no es ocio, eso es vicio.

PU2 16 O: Yo lo que veo es que si tenemos un ocio en Internet pero que para la mayoría de los jóvenes no existe ocio en Internet, sino obsesión en Internet.

Asimismo, consideran que el uso de Internet en el tiempo libre incita al aislamiento. Básicamente, porque comprenden el uso de Internet como un acción físicamente solitaria que motiva quedarse en casa con la única compañía del ordenador y la red.

PU1 16 A: Al final te cierras y te quedas tú sólo. Tú cuando haces extraescolares y así, nestas [sic] con otra gente, te comunicas.

PU1 17 A: Porque para mí Internet no es tan importante, no sé no... Para mí el deporte o las actividades que hago son actividades en las que me relaciono con gente, compañerismo y así... pero Internet al final es un mundo en el que estas tú solo y que por mucho que estés hablando con otras personas, al final estas tú solo y es como que te aíslas del mundo y yo no lo veo así.

\section{E-ocio como distracción y aislamiento}

Por último, concebir el e-ocio como una distracción es otro de los argumentos que adquiere peso entre sus afirmaciones. Los jóvenes opinan que el móvil es una fuente de despiste constante. Un aparato que se posiciona en el centro y que, en ocasiones, incluso es motivo de conflicto. En esta misma línea se sitúa el verbo "procrastinar", esto es, postergar actividades o situaciones que deben atenderse, sustituyéndolas por 
otras situaciones más irrelevantes o agradables. En el caso de los jóvenes nos estaríamos refiriendo a dejar de hacer los deberes o estudiar:

PU2 17 O: Alguna vez sí, es que me doy cuenta pero es que...Yo me doy cuenta porque a veces digo, he pasado toda la tarde, he estado delante del libro y no he pasado ni una página, porque igual estás así, pero de repente te vibra el móvil, sacas tal, estás así y ya como te has distraído pues igual ves una cosas y te pones a ver esa cosa. Además yo que me distraigo muy fácil y que puede estar horas pensando sobre una cosa y estar ahí dándole vueltas, dándole vueltas, aunque sea una tontería...y eso o...

PU1 17 O: No, ipara nada! Al hacer deporte tienes una desconexión que te centras en una cosa y lo das todo ahí y te olvidas del resto, pero en Internet desconectar pero desconectas de tus quehaceres y te centras en otras cosas. Es desconexión más o menos total y en Internet es desconexión de tu vida para hacer otras cosas.

\section{INTERPRETACIÓN DE LOS RESULTADOS DESDE LA PERSPECTIVA EXPERIENCIAL DEL OCIO}

La preocupación por encontrar rasgos comunes en las experiencias de ocio ha sido y continúa es un tema de estudio muy presente. Partiendo de los planteamientos comunes propuestos por Norbert Elias y Eric Dunning (1992) y las reflexiones que a lo largo de los últimos años se han llevado a cabo en el Instituto de Estudios de Ocio (Universidad de Deusto), las siete características más importantes del ocio experiencial son las siguientes (figura 3):

Figura 3. Características del ocio experiencial

El marco de referencia es la persona

Tiene un predominio emocional

Se justifica por la libre satisfacción

Se integra en valores y modos de vida

Tiene un caráter procesual

Se experimenta en distintos grados de intensidad

Requiere formación

Fuente: “Ocio Valioso” (Cuenca, 2014).
Al comparar las prácticas de ocio conectado de los jóvenes con estos rasgos, nos damos cuenta de que, en última instancia, las experiencias de ocio de los jóvenes están relacionadas directamente con las experiencias de e-ocio. Los principales usos que los jóvenes hacen de Internet son estar en redes sociales, comunicarse, usar el WhatsApp, buscar información, chatear y ver vídeos de YouTube, esto es, los aspectos relacional y lúdico continúan siendo factores centrales de su ocio. Lo que nos indica que, en realidad, lo más importante en las experiencias de e-ocio de los jóvenes no es la tecnología en sí misma, sino el relacionarse y divertirse. Las tecnologías digitales actúan como un medio y herramienta que les ayuda a enriquecer sus experiencias de ocio. Por lo tanto, las experiencias de e-ocio actúan como medio para propiciar experiencias de ocio más positivas y gratificantes, es decir, estaríamos hablando de un e-ocio exotélico.

En las experiencias de e-ocio el marco de referencia también es la propia persona. De hecho, una de las principales motivaciones de uso de Internet por la que los jóvenes deciden

conectarse es el factor relacional (comunicarse, conocer a gente curiosear, etc.) que posibilita la red de redes. Además, en el carácter social intrínseco de la red, como comunidad de usuarios, queda implícito el concepto de persona. Independientemente de que sea el usuario de manera aislada o solo desde su casa quien decide conectarse a la red de manera individual, la finalidad es relacionarse con los demás. Un ejemplo de ello es el auge que ha tenido el e-ocio social. Un tipo de ocio fundamentado en el uso constante de las redes sociales de carácter social, valga la redundancia.

En cuanto al predominio emocional, las prácticas de ocio conectado, de la misma manera que el ocio tradicional positivamente valorado por los jóvenes, se asientan en emociones. El e-ocio no se justifica en el deber, y cuando un joven decide conectarse es porque realmente quiere, le agrada y tiene una motivación, en el caso del e-ocio extrínseca, que le impulsa. Asimismo, las experiencias de e-ocio también se integran en valores y modos de vida. El ocio es un valor en sí mismo, pero también un valor subordinado a otros más amplios como la felicidad o la autorrealización de la persona (Cuenca et al., 2012). El ocio positivo potencia valores personales y comunitarios más allá de sí mismo, pero estos valores 
suelen estar relacionados con el tipo de ocio que se practica. Bajo esta premisa podríamos interpretar que la generación de jóvenes interactivos es una generación superficial, ya que el análisis descriptivo del e-ocio deja patente que los jóvenes practican un ocio de carácter más bien trivial.

Dicho de otro modo, el e-ocio de los jóvenes se define por ser un ocio hedonista, placentero, que ofrece recompensas inmediatas y que se fundamenta en el "aquí y ahora", por lo que no es de extrañar que la generación joven, en cierta manera, pueda definirse bajo estos mismos calificativos. "No podemos ser una persona los días laborables y otra los fines de semana” (Cuenca et al., 2012, p. 23). Aspecto que no tiene porque ser negativo, ya que el ocio exotélico también reporta beneficios.

Por otra parte, el e-ocio también transcurre en una temporalidad tridimensional (pasado, presente y futuro), más aún cuando la hipertextualidad, sincronicidad e interactividad propia de la red permite al usuario disfrutar de experiencias en el momento que desea, de igual manera que las experiencias del ocio conectado también pueden experimentarse en distintos niveles de intensidad y ello depende básicamente del uso que se haga de las herramientas digitales. En función de la utilidad que se haga de la red, esto es, como expone Reig (2012), se emplee para informarse y comunicarse (TIC), para aprender y generar conocimiento (TAC) o para empoderarse y participar (TEP), las experiencias de e-ocio serán más o menos valiosas. En nivel de intensidad es un aspecto que tiene relación directa con el nivel de participación de los jóvenes en la red. En este sentido, el e-ocio que definen los jóvenes es de baja intensidad, calificado como "plan b" o "pasa rato".

Por último, esta interpretación del e-ocio de los jóvenes nos muestra que estos no aprovechan lo suficiente las ventajas que ofrece la red lo que, a priori, puede ser un indicio de una falta de formación en materia digital que les eduque en el uso provechos de la red. De hecho, el séptimo rasgo que caracteriza a las experiencias de ocio es la necesidad de una capacitación adecuada.

\section{EN SÍNTESIS}

Vivimos en una nueva era, la era digital. Un nuevo contexto en el que las tecnologías digitales e Internet son protagonistas al influir prácticamente en todos los ámbitos de la vida, entre ellos, el ocio. La esfera del ocio ha notado directamente el impacto de las Tic. Lo que ha provocado una transformación en la manera de experimentarlo.

El ocio propio de la era digital es, valga la redundancia, un ocio digital. Un tipo de ocio que acepta el ocio tradicional digitalizado bajo la influencia de las TIC y promueve nuevos ocios ligados al ciberespacio que necesitan de conexión a Internet para poder ser disfrutados (e-ocio). Estar conectado es hoy una parte esencial en la vida de muchas personas, entre ellos, los jóvenes. Una generación que está creciendo en un contexto social, cultural y educativo distinto, especialmente por la presencia continua y constante de la tecnología, y que presentan rasgos distintivos respecto a otras generaciones.

Los rasgos tecnológicos afectan a todos los ámbitos de su vida, especialmente al referido al ocio que, por otro lado, es un aspecto que en esta edad tiene una importancia vital. Los jóvenes nativos digitales son prosumidores, sociales, multitarea y valoran estar conectados como algo esencial en sus vidas, pero como ya hemos resaltado, ello no significa que sean jóvenes digitalmente competentes. De hecho, las prácticas de e-ocio de los jóvenes son más bien superficiales. Básicamente, emplean las Tic para informarse y comunicarse y apenas como medio y herramienta de aprendizaje y participación.

A través del análisis del discurso de un total de 88 jóvenes, de entre 16 y 18 años de Bizkaia, participantes en nueve grupos de discusión y diez entrevistas en profundidad, se ha constatado que los jóvenes perciben Internet principalmente como un medio y herramienta que les permite relacionarse, comunicarse, informarse y pasar el rato. Lo que nos indica que, en realidad, lo más importante en la experiencia de e-ocio de los jóvenes no es la tecnología en sí misma, sino relacionarse y divertirse. Las tecnologías digitales actúan como un medio que les ayuda a mejorar y enriquecer sus experiencias de ocio tradicionales como son "estar con los amigos", "hacer deporte" y "salir de fiesta" (INJUVE, 2010), de ahí que este se pueda calificar como un ocio exotélico. 


\section{REFERENCIAS}

Bauman, Z. (1999). Modernidad líquida. Buenos Aires: Fondo de Cultura Económica.

Bringué, X., y Sádaba, Ch. (2009). La generación interactiva en España. Niños y adolescentes ante las pantallas. Pamplona: Ariel. Disponible en: http://goo.gl/FlXezM

Castells, M. (1997). La era de la información. Economía, sociedad y cultura (Vol. I, La sociedad red). Madrid: Alianza Editorial.

Castells, M. (2001). Internet y la sociedad red. Madrid: La factoría de Ideas.

Carr, N. (2011). Superficiales ¿Qué está haciendo Internet con nuestras mentes? Madrid: Taurus.

Cuenca, M. (2000). Ocio humanista. Dimensiones y manifestaciones actuales del ocio. Bilbao: Universidad de Deusto.

Cuenca, J. (2008). El ocio líquido: Un nuevo paradigma de experiencia. En M. Monteagudo, La experiencia de ocio: Una mirada científica desde os estudios de ocio. Documentos de Estudios de Ocio, (35), 17-41.

Cuenca, J. (2010). El valor de la experiencia de ocio en la modernidad tardía: Génesis y condiciones de posibilidad (PhD). Bilbao: Universidad de Deusto.

Cuenca, M. (2014). Ocio valioso. Bilbao: Universidad de Deusto.

Cuenca, M., Bayón, F., y Madariaga, A. (2012). Educación y ocio en Vitoria-Gasteiz. Vitoria-Gasteiz : Ayuntamiento de Vitoria-Gasteiz.

Elias, N., y Dunning, E. (1992). Deporte y ocio en el proceso de la civilización. Madrid: Fondo de Cultura Económica.

Feixa, C. (2000). Generación@: la juventud en la era digital. Nómadas, 13, 75-91.

García, E., López, J., y Samper, A. (2012). Retos y tendencias del ocio digital: transformación de dimensiones, experiencias y modelos empresariales. Arbor, 188(754), 395-407.

González, F. (2013). Jóvenes y participación. Estudio sobre participación juvenil. Vitoria: Consejo de la Juventud de Euskadi.

Hwang, S. (2007). Utilizing qualitative data analysis software: A review of ATLAS.ti. Social Science Computer Review, 26(4), 519-527. doi:10.1177/0894439307312485

Horrigan, J. (2009). The mobile difference. Washington: Pew Internet \& American Life Project.
Igarza, R. (2009). Burbujas de ocio: Nuevas formas de consumo cultural. Buenos Aires: La Crujía

Instituto de Juventudes de España (INJUVE) (2012). Sondeo de opinión de la gente joven INJUVE 2011 (1aㅗ encuesta). Madrid: Observatorio de la Juventud en España. Disponible en http://goo.gl/VY50f

Kerckhove, D. (1999). La piel de la cultura. Investigando la nueva realidad electrónica. Barcelona: Gedisa Editorial.

Krueger, R.A. (1991). Focus groups: A practical guide for applied research. Beverly Hills; California: Sage.

Lewis, R. B. (2004). NVivo 2.0 and ATLAS.ti 5.0: A comparative review of two popular qualitative dataanalysis programs. Field Methods, 16(4), 439-464. doi:10.1177/1525822X04269174

Nimrod, G., y Adoni, H. (2012). Conceptualizing eleisure, Loisir et Société. Society and Leisure, 35(1), 31-56.

Observatorio de la Juventud (2012). Informe Juventud 2012 en España. Madrid: INJUVE Disponible en: http://goo.gl/eFGKXl (Última consulta: 22/05/2012)

Observatorio Vasco de la Juventud (Febrero de 2012). Gabinete de Prospección Sociológica. Medios de Comunicación. Disponible en: http://goo.gl/H1wmnA (Última consulta: 16/6/2012)

Observatorio Nacional de las Telecomunicaciones y la Sociedad de la información (ONTSI) (2012). Los contenidos digitales en España. Informe Anual 2011. Madrid.

Prensky, M. (2010). Teaching digital natives: Partnering for real learning. London: Corwin.

Reig, D. (2012). Socionomía: ¿vas a perderte la revolución social? Barcelona: Deusto.

San Salvador del Valle, R. (2009). El tiempo acelerado. El País. Disponible en: ttp://goo.gl/w2ktKu(Última consulta: 02/03/2013)

Small, G., y Vorgan, G. (2008). El cerebro digital. Cómo las nuevas tecnologías están cambiando nuestra mente. Barcelona: Urano.

Tapscott, D. (1998). Creciendo en un entorno digital. Barcelona: McGraw Hill.

Viñals Blanco, A. (2015). Ocio conectado: la experiencia de e-ocio de los jóvenes (16-18 años) de Bizkaia. Bilbao: Universidad de Deusto. 
